\title{
Metastable chimera states in community-structured oscillator networks
}

\author{
Murray Shanahan \\ Department of Computing, Imperial College London, 180 Queen's Gate, London SW7 2AZ, United Kingdom
}

(Received 24 September 2009; accepted 13 January 2010; published online 22 February 2010)

\begin{abstract}
A system of symmetrically coupled identical oscillators with phase lag is presented, which is capable of generating a large repertoire of transient (metastable) "chimera" states in which synchronization and desynchronization coexist. The oscillators are organized into communities, such that each oscillator is connected to all its peers in the same community and to a subset of the oscillators in other communities. Measures are introduced for quantifying metastability, the prevalence of chimera states, and the variety of such states a system generates. By simulation, it is shown that each of these measures is maximized when the phase lag of the model is close, but not equal, to $\pi / 2$. The relevance of the model to a number of fields is briefly discussed with particular emphasis on brain dynamics. () 2010 American Institute of Physics. [doi:10.1063/1.3305451]
\end{abstract}

\begin{abstract}
Many complex systems, both natural and artificial, exhibit synchronization phenomena, which can be modeled using weakly coupled oscillators. Most previous synchronization studies focus on stability. Yet many complex systems (such as the human brain) do not converge on stable synchronized states. Rather they are metastable, temporarily dwelling in the vicinity of one stable state before spontaneously migrating away from it toward another. A second feature of many complex systems (including the brain) is competition. In the context of synchronization, this is manifest in so-called chimera states, where one coalition of oscillators synchronizes while rival coalitions of identical oscillators are desynchronized. This short paper presents the first model to exhibit both chimera states and metastability. This is achieved by organizing the oscillators into a community structured (or modular) network (echoing established brain connectivity findings). Measures are introduced to quantify the prevalence of chimera states and metastability. These form the basis of an empirical study that establishes the conditions in which metastability and chimera states are most prevalent. The same study shows (using a novel measure) that the repertoire of metastable states produced under these conditions is also maximized.
\end{abstract}

\section{INTRODUCTION}

Periodic phenomena involving the synchronization of multiple variables are prevalent both in nature and the human environment, and can be modeled mathematically as systems of coupled oscillators (Pikovsky et al., 2001). Among the rich variety of behaviors such systems exhibit are states in which a set of identical symmetrically coupled oscillators spontaneously partitions into one subset that is synchronized and another subset that is desynchronized (Kuramoto and Battogtokh, 2002; Abrams and Strogatz, 2004; Abrams et al., 2008). A system that gives rise to these so-called chimera states is a plausible model for a competitive process wherein a set of winners forms an alliance to the exclusion of the rest of the population. Since competitive processes of this sort dominate the dynamics of the brain, the economy, and the ecosphere, they are of considerable scientific interest.

Typical studies of synchronization in systems of coupled oscillators attempt to map their various dynamical regimes and to pin down the conditions for entering those regimes (Acebrón et al., 2005). Stable states, in which some or all of the oscillators are fully synchronized, have attracted particular attention. However, in many complex systems, extended periods of synchronization are pathological. Prolonged synchronization in the brain, for example, is a symptom of seizure (Arthuis et al., 2009). This motivates the study of metastability in systems of coupled oscillators (Niebur et al., 1991; Bressler and Kelso, 2001; Pluchino and Rapisarda, 2006; Kitzbichler et al., 2009). A system of oscillators exhibits metastability if some or all of its members linger in the vicinity of a synchronized state without falling into such a state permanently. Moreover, in a complex milieu such as the brain, the economy, or the ecosphere, we should expect to witness a large number of distinct metastable states.

The upshot of these considerations is that an adequate model of competitive periodic phenomena in systems that are not frozen, static, or in seizure should exhibit a nontrivial repertoire of metastable chimera-like states. Although the topic has been relatively neglected, metastability in oscillator networks has been described in the literature before. For example, Niebur et al. (1991) reported metastability in a large network of weakly coupled oscillators. But their model included a noise term, and metastability is promoted by the resulting thermal fluctuations (Kuramoto, 1984, Chap. 5). More recently, Kitzbichler et al. (2009), following the work of Kuramoto (1984), characterized the metastability of a network of oscillators with critical coupling strength. But the natural frequencies of the oscillators in their model are distributed while models of chimera states deploy identical oscillators.

Reinforcing the well-established view that there is a crucial relationship between connectivity and dynamics (Strogatz, 2001; Arenas et al., 2008; Müller-Linow et al., 2008), the required properties are exhibited by the present (deter- 
ministic) model because its oscillators (all identical) are arranged in a network with community structure (Girvan and Newman, 2002). That is to say, the nodes of the network (the individual oscillators) are partitioned into subsets (communities or modules) whose members are more densely connected with each other than with nodes outside their community. Since both the functional and structural connectivity of the human brain are similarly modular (Hagmann et al., 2008), the system of oscillators presented here is a plausible model of metastable neural synchronization.

\section{METHODS AND MEASURES}

The present model comprises eight communities of 32 phase-lagged Kuramoto oscillators with identical natural frequencies (Kuramoto, 1984; Acebrón et al., 2005). Each oscillator is fully connected to its own community and has 32 random connections to oscillators in other communities. Following the model of Abrams et al. (2008), the intracommunity coupling strength is slightly higher than the intercommunity coupling strength. All connections are symmetrical.

The phase $\theta_{i}$ of each oscillator $i$ is governed by the equation

$$
\frac{d \theta_{i}}{d t}=\omega+\frac{1}{N+1} \sum_{j=1}^{N} K_{i, j} \sin \left(\theta_{j}-\theta_{i}-\alpha\right),
$$

where $\omega$ is the natural frequency of the oscillator, $N$ is the total number of connections per oscillator, $K_{i, j}$ is the coupling strength between oscillators $i$ and $j$, and $\alpha$ is a fixed phase lag. In the present model, $\omega=1, N=63, K_{i, j}=u$ if $i$ and $j$ belong to the same community, $K_{i, j}=v$ if $i$ and $j$ belong to different communities and are connected, and $K_{i, j}=0$ otherwise. Like Abrams et al. (2008), we define two parameters $A$ and $\beta$ for the model, such that $A=u-v$, where $u+v=1$, and $\beta=\pi / 2-\alpha$. For the experiment described here $A$ was set at 0.2 .

The level of synchrony within a community $c$ at time $t$ may be quantified according to the measure

$$
\phi_{c}(t)=\left|\left\langle e^{i \theta_{k}(t)}\right\rangle_{k \in c}\right|,
$$

where $\theta_{k}(t)$ is the phase of oscillator $k$ at time $t$ and $\langle f\rangle_{k \in c}$ denotes the average of $f$ over all $k$ in $c$. This measure ranges from 0 to 1 , where 0 is total desynchronization and 1 is full synchronization. [Note that $\phi_{c}(t)$ quantifies instantaneous synchrony and does not provide information about coherence.]

By sampling $\phi_{c}(t)$ for all the communities at discrete intervals, it is possible to quantify both the level of metastability in the system and the prevalence of chimera-like states (Fig. 1). Let $C$ be the set of all $M$ communities and assume $\phi_{c}(t)$ is sampled at times $t \in\{1 \cdots T\}$ for each $c \in C$. If we fix the community $c$ and estimate the variance $\sigma_{\text {met }}(c)$ of $\phi_{c}(t)$ over all time points $t \in\{1 \cdots T\}$, we get an indication of how much the synchrony in $c$ varies over time. The average of this variance estimate over the set $C$ of all communities is an index of the metastability of the overall system (denoted $\lambda$ ). So we have
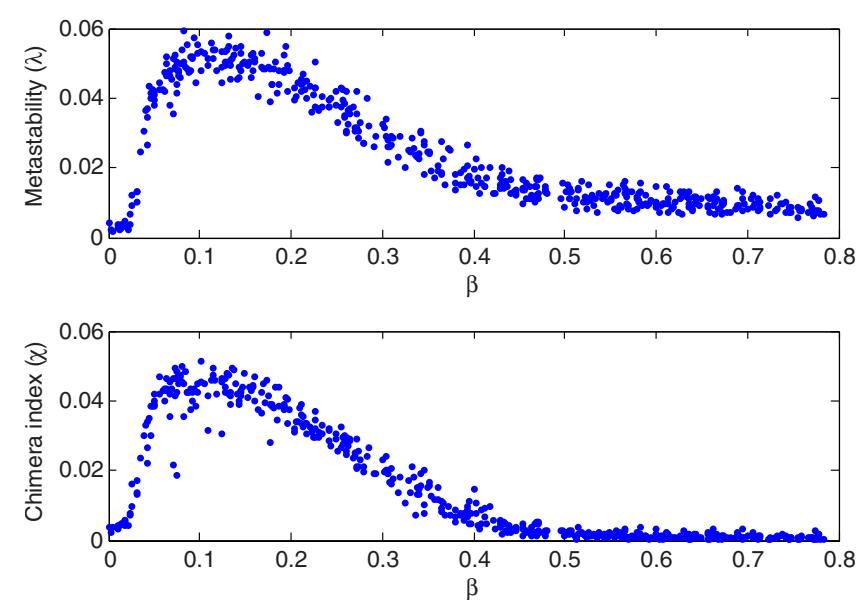

FIG. 1. (Color online) The behavior of the model for randomly generated values of $\beta$ between 0 and $\pi / 4$ over 500 trials. Initial phases were randomized for each trial. Both metastability index $(\lambda)$ and chimera index $(\chi)$ are close to zero for $\beta=0$, peak at around $\beta=0.1$, and tail off rapidly.

$$
\lambda=\left\langle\sigma_{\mathrm{met}}\right\rangle_{C},
$$

where

$$
\sigma_{\text {met }}(c)=\frac{1}{T-1} \sum_{t \leq T}\left(\phi_{c}(t)-\left\langle\phi_{c}\right\rangle_{T}\right)^{2} .
$$

Conversely, if we fix the time $t$ and estimate the variance $\sigma_{\text {chi }}(t)$ of $\phi_{c}(t)$ over all communities in $C$, we get an instantaneous indication of how chimera-like the system is at time $t$. The average of this variance estimate is an index of how chimera-like a typical state of the system is (denoted $\chi$ ). So we have

$$
\chi=\left\langle\sigma_{\text {chi }}\right\rangle_{T},
$$

where

$$
\sigma_{\text {chi }}(t)=\frac{1}{M-1} \sum_{c \in C}\left(\phi_{c}(t)-\langle\phi(t)\rangle_{C}\right)^{2} .
$$

Note that if a population $c$ of oscillators is either completely synchronized or completely desynchronized then $\sigma_{\text {met }}(c)=0$. If $c$ spends equal time in all stages of synchronization then it presents a uniformly distributed $\phi_{c}$ with $\sigma_{\text {met }}(c)=\frac{1}{12} \approx 0.083$. A value of $\sigma_{\text {met }}(c)$ greater than $\frac{1}{12}$ is possible if $\phi_{c}$ has a multimodal distribution with high peaks at both shoulders. But assuming such distributions do not arise, and as long as the population size is large, we may regard a uniform distribution as indicative of maximum metastability, yielding $\lambda_{\text {max }}=\frac{1}{12} \approx 0.083$. [The population size must be large, since, for example, a pair of decoupled (free-running) oscillators with different frequencies is not metastable, yet has uniformly distributed $\phi_{c}$. By contrast, episodes of high synchrony are rare in a large population of decoupled oscillators with different frequencies, ensuring a nonuniformly distributed $\phi_{c}$. In the present model, the community size is large, and of course the oscillators are not decoupled.]

Similar considerations apply to $\sigma_{\text {chi }}$. If at some time $t$ all of the communities in the system are either fully synchronized or fully desynchronized then $\sigma_{\text {chi }}(t)=0$. A "perfect" chimera state might be characterized as one in which exactly 
half of the communities are fully synchronized and half are fully desynchronized [as in the model of Abrams et al. (2008)], which will yield $\sigma_{\text {chi }}(t)=\frac{2}{7} \approx 0.2857$. Although high values of $\sigma_{\text {chi }}$ can be obtained in the present model, due to its metastability they are only transient. So because $\chi$ averages $\sigma_{\text {chi }}$ over time, we should not expect it to approach this maximum. Instead, a metastable system can be considered to attain maximum $\chi$ if it spends half of its time in a maximally chimera-like state and half of its time in a minimally chimera-like state, which yields $\chi_{\max }=\frac{1}{7} \approx 0.1429$.

In addition to assessing synchronization within a community, it is possible to quantify pairwise synchronization across communities. In particular, for every pair of communities $a$ and $b$, we can consider

$$
\psi_{a, b}(t)=\left|\frac{1}{2}\left(\left\langle e^{i \theta_{k}(t)}\right\rangle_{k \in a}+\left\langle e^{i \theta_{k}(t)}\right\rangle_{k \in \in_{b}}\right)\right| .
$$

Note that $\psi_{a, b}(t)$, which ranges from 0 to 1 , will only be high if $\phi_{a}(t)$ and $\phi_{b}(t)$ are individually high and communities $a$ and $b$ are synchronized with each other. If $a$ and $b$ are fully synchronized internally but $180^{\circ}$ out of phase with each other then $\psi_{a, b}(t)=0$.

Although $\lambda$ and $\chi$ taken together can detect the occurrence of metastable chimera states, neither measure can distinguish a system that repeatedly visits the same metastable chimera state from a system that has a large repertoire of metastable chimera states. One way to quantify this repertoire is to assess how "mixed up" is the set of coalitions a system produces over a period of time. Accordingly, we define the (normalized) coalition entropy $H_{C}$ of a system by the equation

$$
H_{C}=-\frac{1}{\log _{2}|S|} \sum_{s \in S} p(s) \log _{2}(p(s)),
$$

where $S$ is the set of distinct coalitions the system can generate and $p(s)$ is the probability of coalition $s$ arising in any given time point. The measure is normalized to lie to between 0 and 1. In the context of the present model we can consider coalitions of synchronized communities. A coalition $s$ is said to arise at time $t$ if $\phi_{c}(t)>\gamma$ for all $c \in s$, where $\gamma$ is a synchronization threshold (we shall use $\gamma=0.8$ ). Clearly for a system of $M$ communities there are $2^{M}$ possible coalitions, so $\log _{2}|S|=M$. If all $2^{M}$ possible coalitions arise with equal probability, we have $H_{C}=1$. On the other hand, if the system resides permanently in the same state (whether fully synchronized, fully desynchronized, or any sort of chimera-like state), then only one coalition arises and we have $H_{C}=0$.

\section{RESULTS}

A series of 1000-step trials of for a range of values of $\beta$ was carried out. Five hundred trials were performed for randomly generated values of $\beta$ ranging from 0 to $\pi / 4$. All numerical simulation was carried out using the fourth-order Runge-Kutta method with a step size of 0.05 . Intracommunity coupling assignments and initial phases were randomized for each trial. The internal synchrony $\phi_{c}(t)$ was calculated for each community at five-step intervals, and the resulting data were used to compute $\lambda, \chi$, and $H_{C}$. In addi-

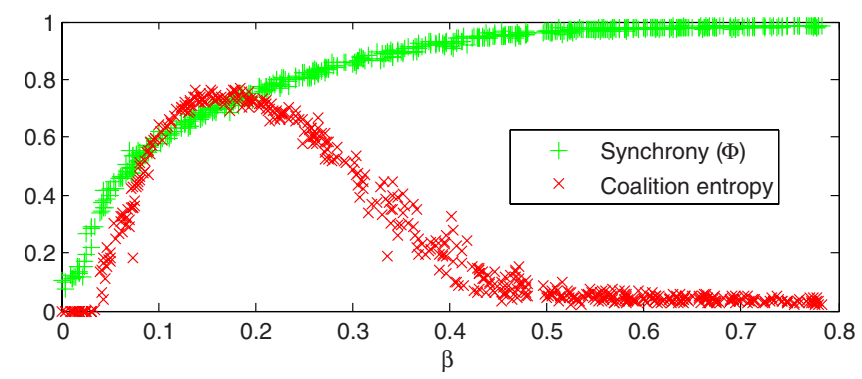

FIG. 2. (Color online) Global synchrony $(\Phi)$ and coalition entropy for the set of trials depicted in Fig. 1. Coalition entropy presents a similar profile to metastability and chimera index (Fig. 1), although it peaks slightly later at around $\beta=0.15$.

tion, an index of global synchrony $\Psi$ was calculated, taken as the average of $\phi_{c}(t)$ over all times and communities.

The results are presented in scatter plots of Figs. 1 and 2. These figures suggest that the model behaves as advertised, and is capable of generating a large repertoire of metastable chimera-like states, but only when $\beta$ falls within a certain narrow range. Metastability and chimera indices are maximized when $0.05<\beta<0.15$ (Fig. 1), at which point $0.6<\Psi<0.7$ (Fig. 2). When $\beta=0$ (i.e., the phase lag is exactly $\pi / 2$ ), the system finds it hard to synchronize at all, and each of the measures is correspondingly low. At the other end of the scale, when $\beta>\pi / 8$, the system tends toward full synchronization in all communities, and metastability, chimera index, and coalition entropy all tail off accordingly. Coalition entropy peaks slightly later than the other two measures with $0.1<\beta<0.2$ (Fig. 2).

As with other systems of oscillators that exhibit metastability, fluctuations of synchronization and desynchronization are prevalent only in a narrow, critical region that resembles a thermodynamic phase transition from order to disorder (Kuramoto, 1984, Chap. 5). The area to the left of the critical region is the disordered regime, wherein the phases of the oscillators are subject to predominantly repelling forces, while the area to the right of the critical region is the ordered regime, wherein attracting forces dominate. In the critical region there is a balance of repelling and attracting forces. But this balance is not static, and the dominant force applicable to each community alternates between phase attraction and phase repulsion. Since the oscillators have identical natural frequencies and there is no external stochastic perturbation, the reasons for this are not clear, and further work is required to understand the mechanisms underlying the model's behavior.

To gain some preliminary insight into the behavior of the model in the critical region, we shall examine a single run in detail. Figure 3(a) shows the evolution of synchrony $(\phi)$ within all eight oscillator communities over a 200 time-step interval in a typical trial with $\beta \approx 0.1$. The trial is representative of the dynamical regime of most interest to us here. The statistics for this trial are $\chi=0.0525, \lambda=0.0542$, $H_{C}=0.6341$, and $\Phi=0.5798$. The system exhibits several chimera-like states in which some oscillator communities are highly synchronized while others are desynchronized. From time 250 to 270 , for example, three communities are highly 

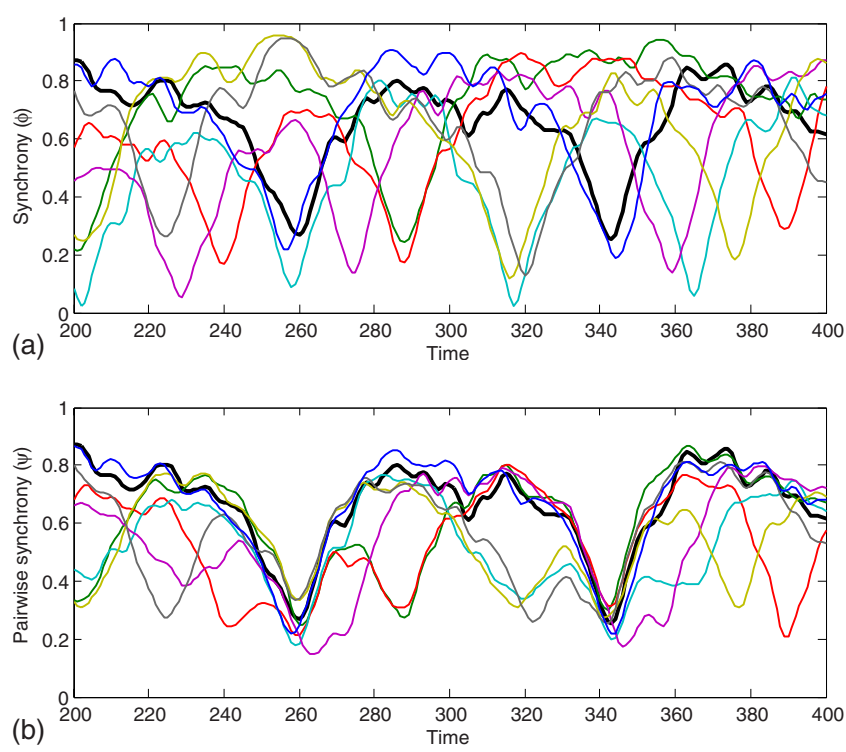

FIG. 3. (Color online) A 200 time-step period in a typical run with $\beta \approx 0.1$. (a) Intracommunity synchrony: the system exhibits several clear chimeralike states. From time 250 to 270, for example, three communities are highly synchronized and three are desynchronized. (b) Intercommunity synchrony: pairwise synchrony is plotted between one selected community (shown in black) and each of the eight communities (including itself). From time 310 to 330 the selected community is synchronized with several others, forming a temporary coalition.

synchronized $(\phi>0.8)$ and three are desynchronized $(\phi<0.4)$, while two communities have intermediate values of $\phi$. A similarly clear chimera-like state can be seen from time 310 to 330 , but with a different combination of synchronized communities.

Figure 4 shows how synchrony is distributed over time for each of the eight communities in the same sample run. As expected from Fig. 3(a), none of the communities spends the majority of its time in any one stage of internal synchronization. Despite a tendency toward synchrony (a rightward skew), the resulting distributions all have high variance. It is noteworthy, however, that different communities exhibit different profiles, with community 2 , for example, showing a
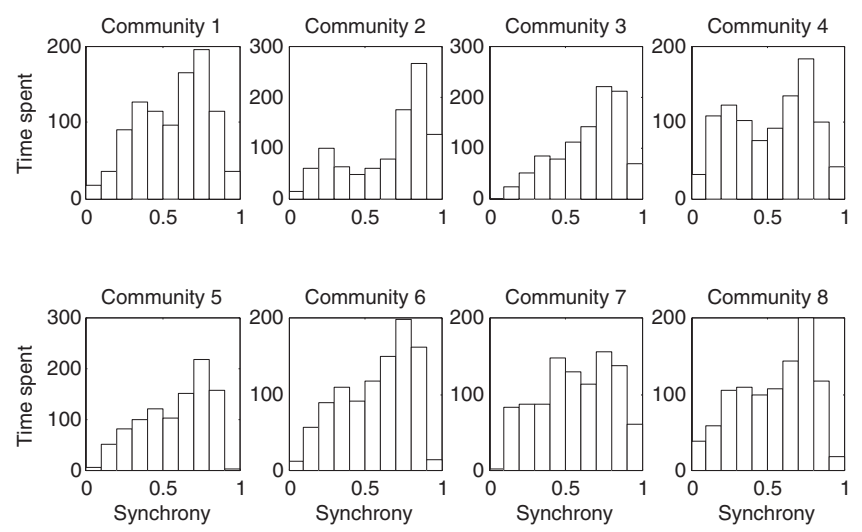

FIG. 4. The distribution of $\phi$ for each community in the run depicted in Fig. 3. The distributions all have variances far from zero, indicating metastability in the sense that the oscillator communities spend time in all stages of synchronization. more pronounced tendency to internally synchronize than its peers.

The plots in Fig. 3(a) are highly irregular, and there is no clear pattern to the coincidence of peaks and troughs, which suggests that the system is capable of generating a large repertoire of coalitions of simultaneously synchronized communities. By contrast, the equivalent plot for a "breathing" chimera state has a regular rhythm [see Fig. 2 of Abrams et al. (2008)]. The lack of any discernible regularity in the sequence of metastable states visited by the system hints at both chaotic itinerancy (Kaneko and Tsuda, 2003; Tsuda et al., 2004) and dynamical complexity (Tononi et al., 1998; Seth et al., 2006; Shanahan, 2008b), but further work would be required to establish these properties rigorously.

Further insight into the generation of coalitions is gained by examining the ebb and flow of pairwise synchronization $(\psi)$. Figure 3(b) shows, for the same 200-step period of the same trial, the pairwise synchrony $\psi_{a, b}$ between a selected community $a$ and all communities $b$ (including $a$ itself). As noted in Sec. II, the pairwise synchronization between two oscillator communities will be low if either community has low internal synchronization. In the troughs near times 260 and 340, all pairwise synchrony measures are low because the internal synchrony of community $a$ is itself low. At other times, the internal synchrony of $a$ is high, as is the pairwise synchrony between $a$ and various peers. Sets of such synchronized peers constitute temporary coalitions, whose constitution varies over time. At time 290, for example, $a$ is in a coalition alongside five of its peers, with two communities excluded. But by time 320 three communities have been expelled from the coalition, while the two previous exclusions have been recruited into its membership.

\section{DISCUSSION}

Although the essential characteristic of the model-the ability to generate a large repertoire of metastable chimera states-reflects properties common to many real-world complex dynamical systems, the task remains of mapping each of those systems onto the model. For example, it has been proposed that synchronized oscillations in the brain permit effective cooperation among distinct populations of neurons (Fries, 2005, 2009; Womelsdorf et al., 2007), while phenomena such as binocular rivalry, inattentional blindness, and the Stroop effect attest to their competitive character. In other words, the dynamics of the brain seems to arise from the interplay of cooperation and competition, resulting in the formation of synchronized coalitions (Doesburg et al., 2009). Moreover, thanks to an animal's ongoing activity, its brain is endlessly subject to an open-ended variety of perturbations, and to respond effectively these coalitions must be in constant flux. The dynamics of the present model exhibits the same combination of features. However, to date there is no neurologically detailed model to match, although several existing spiking neuron models deal with relevant issues, such as cortical competition (Dehaene et al., 2003; Deco and Rolls, 2005; Shanahan, 2008a), community structure (Shanahan, 2008b), and the interplay of synchronization and desynchronization (Tsuda et al., 2004). 
The findings reported here raise a number of questions to be addressed in future work. The issues of chaotic itinerancy and dynamical complexity have already been mentioned. Another urgent task is to develop a theoretical understanding of the phenomena described. The obvious starting point is the analytical treatment of Abrams et al. (2008), whose model is the basis for the present work. However, analogous results may be difficult to obtain for the present model, given its more complex network structure and the variety of synchronization effects it displays. Finally, it would be fruitful to investigate the occurrence of metastable chimera states in networks with hierarchical community structure [especially as brain networks exhibit this property (Zhou et al., 2006; Ferrarini et al., 2009)], and to attempt to characterize the "path" to such states in the manner of Arenas et al. (2006).

Abrams, D. M., Mirollo, R., Strogatz, S. H., and Wiley, D. A., "Solvable model for chimera states of coupled oscillators," Phys. Rev. Lett. 101, 084103 (2008)

Abrams, D. M. and Strogatz, S. H., "Chimera states for coupled oscillators," Phys. Rev. Lett. 93, 174102 (2004).

Acebrón, J. A., Bonilla, L. L., Vicente, C. J. P., Ritort, F., and Spigler, R., "The Kuramoto model: A simple paradigm for synchronization phenomena," Rev. Mod. Phys. 77, 137-185 (2005).

Arenas, A., Díaz-Guilera, A., Kurths, J., Moreno, Y., and Zhao, C., "Synchronization in complex networks," Phys. Rep. 469, 93-153 (2008).

Arenas, A., Díaz-Guilera, A., and Pérez-Vicente, C. J., "Synchronization reveals topological scales in complex networks," Phys. Rev. Lett. 96, 114102 (2006)

Arthuis, M., Valton, L., Régis, J., Chauvel, P., Wendling, F., Naccache, L., Bernard, C., and Bartolomei, F., "Impaired consciousness during temporal lobe seizures is related to increased long-distance cortical-subcortical synchronization," Brain 132, 2091-2101 (2009).

Bressler, S. L. and Kelso, J. A. S., "Cortical coordination dynamics and cognition," Trends Cogn. Sci. 5, 26-36 (2001).

Deco, G. and Rolls, E. T., "Neurodynamics of biased competition and cooperation for attention: A model with spiking neurons," J. Neurophysiol. 94, 295-313 (2005).

Dehaene, S., Sergent, C., and Changeux, J.-P., "A neuronal network model linking subjective reports and objective physiological data during conscious perception," Proc. Natl. Acad. Sci. U.S.A. 100, 8520-8525 (2003).

Doesburg, S. M., Green, J. J., McDonald, J. J., and Ward, L. M., "Rhythms of consciousness: Binocular rivalry reveals large-scale oscillatory network dynamics mediating visual perception," PLoS ONE 4, e6142 (2009).

Ferrarini, L., Veer, I. M., Baerends, E., van Tol, M.-J., Renken, R. J., van der Wee, N. J. A., Veltman, D. J., Aleman, A., Zitman, F. G., Penninx, B. W. J. H., van Buchem, M. A., Reiber, J. H. C., Rombouts, S. A. R. B., and
Milles, J., "Hierarchical functional modularity in the resting-state human brain," Hum. Brain Mapp 30, 2220-2231 (2009).

Fries, P., "A mechanism for cognitive dynamics: Neuronal communication through neuronal coherence," Trends Cogn. Sci. 9, 474-480 (2005).

Fries, P., "Neuronal gamma-band synchronization as a fundamental process in cortical computation," Annu. Rev. Neurosci. 32, 209-224 (2009).

Girvan, M. and Newman, M. E. J., "Community structure in social and biological networks," Proc. Natl. Acad. Sci. U.S.A. 99, 7821-7826 (2002)

Hagmann, P., Cammoun, L., Gigandet, X., Meuli, R., Honey, C. J., Wedeen, V. J., and Sporns, O., "Mapping the structural core of human cerebral cortex," PLoS Biol. 6, e159 (2008).

Kaneko, K. and Tsuda, I., "Chaotic itinerancy," Chaos 13, 926-936 (2003).

Kitzbichler, M. G., Smith, M. L., Christensen, S. R., and Bullmore, E., "Broadband criticality of human brain network synchronization," PLOS Comput. Biol. 5, e1000314 (2009).

Kuramoto, Y., Chemical Oscillations, Waves, and Turbulence (SpringerVerlag, Berlin, 1984).

Kuramoto, Y. and Battogtokh, D., "Coexistence of coherence and incoherence in nonlocally coupled phase oscillators," Nonlinear Phenom. Complex Syst. (Dordrecht, Neth.) 5, 380-385 (2002).

Müller-Linow, M., Hilgetag, C. C., and Hütt, M.-T., "Organization of excitable dynamics in hierarchical biological networks," PLOS Comput. Biol. 4, e1000190 (2008)

Niebur, E., Schuster, H. G., and Kammen, D. M., "Collective frequencies and metastability in networks of limit-cycle oscillators with time delay," Phys. Rev. Lett. 67, 2753-2756 (1991).

Pikovsky, A., Rosenblum, M., and Kurths, J., Synchronization: A Universal Concept in Nonlinear Sciences (Cambridge University Press, Cambridge, 2001).

Pluchino, A. and Rapisarda, A., "Metastability in the Hamiltonian mean field model and Kuramoto model," Physica A 365, 184-189 (2006).

Seth, A. K., Izhikevich, E. M., Reeke, G. N., and Edelman, G. M., "Theories and measures of consciousness: An extended framework," Proc. Natl. Acad. Sci. U.S.A. 103, 10799-10804 (2006).

Shanahan, M., "A spiking neuron model of cortical broadcast and competition," Conscious Cogn 17, 288-303 (2008a).

Shanahan, M., "Dynamical complexity in small-world networks of spiking neurons," Phys. Rev. E 78, 041924 (2008b).

Strogatz, S. H., "Exploring complex networks," Nature (London) 410, 268 276 (2001).

Tononi, G., Edelman, G. M., and Sporns, O., "Complexity and coherency: Integrating information in the brain," Trends Cogn. Sci. 2, 474-484 (1998)

Tsuda, I., Fuji, H., Tadokoro, S., Yasuoka, T., and Yamaguti, Y., "Chaotic itinerancy as a mechanism of irregular changes between synchronization and desynchronization in a neural network," J. Integr. Neurosci. 3, 159182 (2004).

Womelsdorf, T., Schoffelen, J.-M., Oostenveld, R., Singer, W., Desimone, R., Engel, A. K., and Fries, P., "Modulation of neuronal interactions through neuronal synchronization," Science 316, 1609-1612 (2007).

Zhou, C., Zemanová, L., Zamora, G., Hilgetag, C. C., and Kurths, J., "Hierarchical organization unveiled by functional connectivity in complex brain networks," Phys. Rev. Lett. 97, 238103 (2006). 